\title{
Dermatopathology in the Molecular Age
}

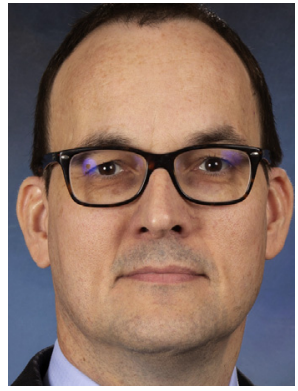

Michael T. Tetzlaff, MD, PhD Editor

The field of dermatopathology remains one of the most dynamic subspecialties in all of pathology. Accurate diagnoses typically require a systematic integration of histopathologic features together with clinical, immunohistochemical, and molecular findings. As the arsenal of molecular studies applied to skin lesions expands, the complexity of this integration increases. In this issue, we assembled leaders across the spectrum of dermatopathology to provide comprehensive reviews and updates on various topics in the field. Melanocytic lesions are emphasized at multiple points, including a historical overview of dysplastic nevi as well as an update on PRAME immunohistochemistry and applications of this emerging and useful marker. Recent studies on mucosal melanomas identifying histopathologic prognostic biomarkers and molecular drivers are reviewed herein. Finally, there is a comprehensive historical overview and contemporary update on the molecular genetics of pigmented epithelioid melanocytoma. The diagnosis of adnexal tumors similarly remains a formidable challenge, as there can be considerable histopathologic overlap among them. The application of nextgeneration sequencing studies has changed this field altogether, so a comprehensive update on molecular genetic advances across the spectrum of adnexal tumors is provided. Given their unique biology, an additional article specifically focuses on the molecular genetic features of sebaceous neoplasia. Molecular advances in soft tissue tumors continue to refine their classification, so an article emphasizing new entities in cutaneous soft tissue tumors is provided. There is also a comprehensive update on cutaneous lymphomas as the complexity of these continues to evolve. Similarly, a review of nail pathologic condition contextualizes a rapidly developing area of dermatopathology. Dermatopathology also requires an intricate understanding of inflammatory diseases, and as such, comprehensive overviews of cutaneous vasculitis and connective tissue diseases, including novel biomarkers and molecular studies identifying critical drivers of these processes, are provided. Finally, with the success of immune modulatory agents in the treatment of cancer, the myriad of cutaneous toxicities caused by these agents continues to grow, and a comprehensive update on "oncodermatopathology" is also provided. These updates are written by experts in their respective topic, each of whom is a practicing dermatopathologist who recognizes the salient points that inform and improve the practice of dermatopathology.

Michael T. Tetzlaff, MD, PhD

Dermatopathology and Oral Pathology Unit The University of California, San Francisco 1701 Divisadero Street, \#280 San Francisco, CA 94115, USA

E-mail address: michael.tetzlaff@ucsf.edu 